\title{
Editors' Choice in the 60th Anniversary Year of Thrombosis and Haemostasis: Past, Present and Future
}

\author{
Christian Weber ${ }^{1}$ Gregory Y. H. Lip ${ }^{2}$ \\ ${ }^{1}$ Institute for Cardiovascular Prevention, Ludwig-Maximilians- \\ University, Munich, Germany \\ 2 Haemostasis Thrombosis and Vascular Biology Unit, City Hospital, \\ Birmingham, West Midland, United Kingdom
}

Thromb Haemost 2018;118:225-227.

2017 marks a very special year for Thrombosis and Haemostasis: it marked the 60th anniversary of the journal. To commemorate this event, selected section editors were invited to create a modern portrait of the journal, reflecting the milestones of the past and encouraging a glance into future across the many topics of the journal's scope. The main directions reflected in the anniversary editorial ${ }^{1}$ are recapitulated in this year's Editors' Choice selection of papers of high interest or citation.

We traditionally start the New Near with various position and consensus papers and current state-of-the-art developments, revealing valuable insights for researchers and clinical specialists. For instance, the role of microvesicles in the pathophysiology of thrombosis and cardiovascular disease has been intensely investigated in light of their value as potential biomarkers. We are therefore delighted to publish a relevant position paper of the European Society of Cardiology Working Group on Atherosclerosis and Vascular Biology, which comprehensively summarizes the current mechanistic knowledge on the formation, composition and function of microvesicles with an endothelial, platelet, red blood cell and leukocyte origin. ${ }^{2}$

In addition, we also published an executive summary of a joint consensus document from the European Heart Rhythm Association and European Society of Cardiology Working Group on Thrombosis, endorsed by the ESC Working Group on Valvular Heart Disease, Cardiac Arrhythmia Society of Southern Africa (CASSA), Heart Rhythm Society (HRS), Asia Pacific Heart Rhythm Society (APHRS), South African Heart (SA Heart) Association and Sociedad Latinoamericana de Estimulación Cardíaca y Electrofisiología (SOLEACE), addressing the topic of antithrombotic therapy in atrial fibrillation (AF) associated with valvular heart disease. ${ }^{3}$ This document recommends that the term 'valvular AF' is outdated and proposes

\author{
Address for correspondence Christian Weber, MD, Institute for \\ Cardiovascular Prevention, Ludwig-Maximilians-University, \\ Pettenkoferstraße 9, Munich 80336, Germany \\ (e-mail: christian.weber@med.uni-muenchen.de).
}

a new functional Evaluated Heartvalves, Rheumatic or Artificial (EHRA) categorization in relation to the type of oral anticoagulant use in patients with AF, as follows: (1) EHRA Type 1 valvular heart disease which refers to AF patients with 'valvular heart disease needing therapy with a vitamin $K$ antagonist (VKA)' and (2) EHRA Type 2 valvular heart disease, which refers to AF patients with 'valvular heart disease needing therapy with a VKA or a non-VKA oral anticoagulant (NOAC)', also taking into consideration score risk factors.

\section{New Developments in Anticoagulants}

Anticoagulants are the mainstay for the prevention and treatment of thrombosis. In their joint paper, Weitz and Harenberg highlighted the advances of oral anticoagulant therapy, referring to safety issues and reviewing some of the ongoing trials with NOACs, and provided insights into recent research on factors XII and XI inhibitors as potentially novel anticoagulants. ${ }^{4}$

Poterucha et al in their review focused on heparin interference in the inflammatory pathophysiology of thrombosis development and tended to advancing treatments based on heparin derivatives. ${ }^{5}$ To complement this, Schulman et al reviewed published as well as ongoing observational studies with NOACs in VTE and discussed limitations in analysis and interpretation. $^{6}$

With the NOACs, the quest for reversal agents continues, and there was much interest in the paper by Ansell et $\mathrm{al}^{7}$ on ciraparantag, a new 'universal antidote' and small molecule that potentially reverses the anticoagulant effects of dabigatran and factor Xa inhibitors.

However, despite the developments with NOACs, the VKAs are not 'dead' and we published interesting papers on the pharmacology of tecarfarin, a novel VKA drug. ${ }^{8,9}$ This complements the clinical data from the EmbraceAC trial. ${ }^{10}$ received

November 29, 2017

accepted

November 29, 2017
Copyright @ 2018 Schattauer

DOI https://doi.org/ 10.1160/TH17-11-0844. ISSN 0340-6245. 


\section{Venous Thromboembolism}

Venous thromboembolism (VTE) as the third most frequent acute cardiovascular syndrome is associated with a considerable disease burden. In the anniversary issue, Schulman et al recalled therapeutic developments focusing on prophylaxis, attending to new drugs and technical advances in imaging, as well as hospital accreditation and funding based on evidence-based practice. ${ }^{11}$

Additional analyses from the large trials included a pooled analysis of RE-COVER and RE-COVER II trials comparing dabigatran versus warfarin for acute venous thromboembolism in elderly or impaired renal function patients. ${ }^{12}$

Apart from clinical trials, real-world observational data on NOACs are increasingly evident. As one notable example, Coleman et al assessed the effectiveness and safety of rivaroxaban versus warfarin in VTE patients in routine practice and came to the conclusion that it more effectively reduces patients' hazard of both recurrent VTE and major bleeding. ${ }^{13}$ Trujillo-Santos et al investigated the use of NOACs under real-life conditions, including nonrecommended doses or regimens and unveiled the influence on VTE occurrence and subsequent outcomes. ${ }^{14}$ We also published the design paper for RE-COVERY DVT/PE, a prospective observational study of acute venous thromboembolism with a focus on dabigatran etexilate. $^{15}$

\section{Metabolic-Vascular Disease}

Endothelial cell metabolism has emerged as an important regulator of angiogenesis. In their review, Breier et al provided insights into angiogenesis during obesity-related metabolic dysfunction and into signaling pathways linking cell metabolism to endothelial function. ${ }^{16} \mathrm{~A}$ set of publications highlighted the impact of hepatic alterations on thrombosis. Ambrosino et al figured out the importance of specific VTE prevention strategies for cirrhotic subjects, ${ }^{17}$ whereas an interesting contribution to elucidating the molecular and pathogenic mechanisms underlying nonalcoholic steatohepatitis (NASH) was provided by Geys et al, demonstrating that ADAMTS13 deficiency in obese mice induces hepatic microthrombosis. ${ }^{18,19}$ Lallukka et al demonstrated that obesity and insulin resistance rather than liver fat increases circulating coagulation factor activities. ${ }^{20}$ Finally, adipose tissue inflammation starts with cell accumulation. Having disclosed a role of leukocyte integrin Mac-1 in macrophage accumulation in adipose tissue in a murine model, Wolf et $\mathrm{al}^{21}$ remained sceptical concerning the net effect of integrin blockade in cardiometabolic disease.

\section{Stroke Prevention in Atrial Fibrillation}

The evolution of stroke and bleeding risk assessment over the past decades was reviewed by Lip et al, ${ }^{22}$ culminating in a practical management pathway to help streamline and simplify decision making for stroke prevention in patients with AF with three simple steps and to aid decision making for stroke prevention in AF (referred to as the Birmingham '3-step').
Biomarker scores represent an attractive prognostic tool for bleeding risk assessment. The performance of the new ABCbleeding score was validated and compared with the HAS-BLED score in a 'real-world' setting of anticoagulated AF patients with long-term follow-up, which concluded that HAS-BLED performed significantly better in predicting major bleeding. ${ }^{23}$ In the largest 'real-world' study on apixaban effectiveness and safety to date, Li et al showed that apixaban significantly attenuates stroke risk and major bleeding compared with warfarin independent from risk subgroups and dose regimens. ${ }^{24}$

\section{Therapeutic Strategies for Atherosclerosis and Atherothrombosis}

Atherosclerosis and atherothrombosis continue to be the leading causes of death worldwide. In their review, Weber et $\mathrm{al}^{25}$ provided historical overview, spotted the range of currently available state-of-the-art therapies and highlighted the promising therapeutic strategies aimed at reduction of the residual risk that still persists despite current therapeutic options. Jamasbi et al underscored their expectations for novel antiplatelet drugs selectively inhibiting arterial thrombosis without interfering with normal haemostasis. ${ }^{26}$

The nature of atherosclerosis had been extensively studied through sophisticated transgenic animal models. As an example, Winkels et al revealed the new atherosclerosismodulating properties of CD70 through altering macrophage function. ${ }^{27}$ Pointing out the recent failures in translating various anti-inflammatory therapeutic strategies for use in humans demands to retain a healthy scepticism regarding the inflammatory causality underlying human atherosclerosis despite the recent success of the CANTOS trial, Santovito and Weber advised to keep in mind that 'things may not always be what they appear. ${ }^{28}$

\section{Antithrombotic Therapy for Acute Coronary Syndrome}

Plaque erosions and ruptures accompany arterial thrombus formation in the coronary arteries resulting in acute coronary syndrome. Sibbing et al highlighted a discrepancy between current guideline recommendations favouring potent platelet inhibition in ACS and the utilization of the respective drugs in clinical practice and pointed out the development of optimized antiplatelet treatment strategies and their utilization in the real world. ${ }^{29}$ Standard care for treating non-ST elevation myocardial infarction patients is represented by dual antiplatelet therapy. Interestingly, chewing versus an equal dose of traditional oral administration enhances inhibition of platelet aggregation after administering a ticagrelor. ${ }^{30}$

\section{Beyond Cardiovascular Disease}

The biochemical characterization of the proteolytic pathways that constitute blood coagulation is followed by identifying and validating appropriate targets for improving global health through their application in haemostasis and thrombosis pathologies. In their review, Ten Cate et al evaluated the 
concepts providing a modern vision of coagulation, illustrating the importance of the coagulation cascade in cardiovascular pathology through thrombotic as well as atherosclerotic processes and in the response to ischaemia-reperfusion injury. ${ }^{31}$ Severe inflammatory complications often determine the disease progression and outcome. The evidence from both basic research and clinical studies highlighted the role of selfextracellular nucleic acids in the crosstalk between immunity and cardiovascular diseases. Preissner et al delivered a portrait of self-extracellular nucleic acids with an emphasis on their role in immune response, inflammation, thrombosis and cardiovascular diseases. ${ }^{32}$

Over the past 60 years, Thrombosis and Haemostasis continues be a platform for exciting developments in vascular biology and medicine, welcoming next generation of authors with novel and amazing approaches in all aspects of coagulation, haemostatic and vascular research. We look forward to help you in sharing and disseminating your knowledge.

\section{References}

1 Weber C, Lip GYH. Thrombosis and Haemostasis: past, present and future. Thromb Haemost 2017;117:1217-1218

2 Ridger VC, Boulanger CM, Angelillo-Scherrer A, et al; Position Paper of the European Society of Cardiology (ESC) Working Group on Atherosclerosis and Vascular Biology. Microvesicles in vascular homeostasis and diseases. Thromb Haemost 2017;117(07): 1296-1316

3 Lip GYH, Collet JP, de Caterina R, et al. Thromb Haemost 2017;117 (12):2215-2236. https://doi.org/10.1160/TH-17-10-0709

4 Weitz JI, Harenberg J. New developments in anticoagulants: past, present and future. Thromb Haemost 2017;117(07):1283-1288

5 Poterucha TJ, Libby P, Goldhaber SZ. More than an anticoagulant: Do heparins have direct anti-inflammatory effects? Thromb Haemost 2017;117(03):437-444

6 Schulman S, Singer D, Ageno W, Casella IB, Desch M, Goldhaber SZ. NOACs for treatment of venous thromboembolism in clinical practice. Thromb Haemost 2017;117(07):1317-1325

7 Ansell JE, Bakhru SH, Laulicht BE, et al. Single-dose ciraparantag safely and completely reverses anticoagulant effects of edoxaban. Thromb Haemost 2017;117(02):238-245

8 Albrecht D, Turakhia MP, Ries D, et al. Pharmacokinetics of tecafarin and warfarin in patients with severe chronic kidney disease. Thromb Haemost 2017;117(11):2026-2033

9 Albrecht D, Ellis D, Canafax DM, et al. Pharmacokinetics and pharmacodynamics of tecarfarin, a novel vitamin $\mathrm{K}$ antagonist oral anticoagulant. Thromb Haemost 2017;117(04):706-717

10 Whitlock RP, Fordyce CB, Midei MG, et al. A randomised, double blind comparison of tecarfarin, a novel vitamin K antagonist, with warfarin. The EmbraceAC Trial. Thromb Haemost 2016;116(02): 241-250

11 Schulman S, Ageno W, Konstantinides SV. Venous thromboembolism: past, present and future. Thromb Haemost 2017;117(07): 1219-1229

12 Goldhaber SZ, Schulman S, Eriksson H, et al. Dabigatran versus warfarin for acute venous thromboembolism in elderly or impaired renal function patients: pooled analysis of RE-COVER and RE-COVER II. Thromb Haemost 2017;117(11):2045-2052

13 Coleman CI, Bunz TJ, Turpie AGG. Effectiveness and safety of rivaroxaban versus warfarin for treatment and prevention of recurrence of venous thromboembolism. Thromb Haemost 2017;117(10):1841-1847
14 Trujillo-Santos J, Di Micco P, Dentali F, et al; RIETE Investigators. Real-life treatment of venous thromboembolism with direct oral anticoagulants: the influence of recommended dosing and regimens. Thromb Haemost 2017;117(02):382-389

15 Ageno W, Casella IB, Han CK, et al. RE-COVERY DVT/PE: Rationale and design of a prospective observational study of acute venous thromboembolism with a focus on dabigatran etexilate. Thromb Haemost 2017;117(02):415-421

16 Breier G, Chavakis T, Hirsch E. Angiogenesis in metabolic-vascular disease. Thromb Haemost 2017;117(07):1289-1295

17 Ambrosino P, Tarantino L, Di Minno G, et al. The risk of venous thromboembolism in patients with cirrhosis. A systematic review and meta-analysis. Thromb Haemost 2017;117(01):139-148

18 Geys L, Bauters D, Roose E, et al. ADAMTS13 deficiency promotes microthrombosis in a murine model of diet-induced liver steatosis. Thromb Haemost 2017;117(01):19-26

19 Wojta J. What ADAMTS13 does in the liver.... Thromb Haemost 2017;117(01):6

20 Lallukka S, Luukkonen PK, Zhou Y, et al. Obesity/insulin resistance rather than liver fat increases coagulation factor activities and expression in humans. Thromb Haemost 2017;117(02):286-294

21 Wolf D, Bukosza N, Engel D, et al. Inflammation, but not recruitment, of adipose tissue macrophages requires signalling through Mac-1 (CD11b/CD18) in diet-induced obesity (DIO). Thromb Haemost 2017;117(02):325-338

22 Lip G, Freedman B, De Caterina R, Potpara TS. Stroke prevention in atrial fibrillation: past, present and future. Comparing the guidelines and practical decision-making. Thromb Haemost 2017;117 (07):1230-1239

23 Esteve-Pastor MA, Rivera-Caravaca JM, Roldan V, et al. Long-term bleeding risk prediction in 'real world' patients with atrial fibrillation: comparison of the HAS-BLED and ABC-Bleeding risk scores. The Murcia Atrial Fibrillation Project. Thromb Haemost 2017;117(10):1848-1858

24 Li XS, Deitelzweig S, Keshishian A, et al. Effectiveness and safety of apixaban versus warfarin in non-valvular atrial fibrillation patients in "real-world" clinical practice. A propensity-matched analysis of 76,940 patients. Thromb Haemost 2017;117(06): $1072-1082$

25 Weber C, Badimon L, Mach F, van der Vorst EPC. Therapeutic strategies for atherosclerosis and atherothrombosis: Past, present and future. Thromb Haemost 2017;117(07):1258-1264

26 Jamasbi J, Ayabe K, Goto S, Nieswandt B, Peter K, Siess W. Platelet receptors as therapeutic targets: past, present and future. Thromb Haemost 2017;117(07):1249-1257

27 Winkels H, Meiler S, Smeets E, et al. CD70 limits atherosclerosis and promotes macrophage function. Thromb Haemost 2017;117 (01):164-175

28 Santovito D, Weber C. Atherosclerosis revisited from a clinical perspective: still an inflammatory disease? Thromb Haemost 2017;117(02):231-237

29 Sibbing D, Angiolillo DJ, Huber K. Antithrombotic therapy for acute coronary syndrome: past, present and future. Thromb Haemost 2017;117(07):1240-1248

30 Asher E, Frydman S, Katz M, et al; For The PLATIS (Platelets and Thrombosis in Sheba) Study Group. Chewing versus swallowing ticagrelor to accelerate platelet inhibition in acute coronary syndrome - the CHEERS study. Thromb Haemost 2017;117(04): 727-733

31 Ten Cate H, Hackeng TM, García de Frutos P. Coagulation factor and protease pathways in thrombosis and cardiovascular disease. Thromb Haemost 2017;117(07):1265-1271

32 Preissner KT, Herwald H. Extracellular nucleic acids in immunity and cardiovascular responses: between alert and disease. Thromb Haemost 2017;117(07):1272-1282 